\title{
An approach for cross-modality guided quality enhancement of liver image
}

\author{
Ahmed Elaraby ${ }^{1}$, Ayman Taha ${ }^{2}$ \\ ${ }^{1}$ Department of Computer Science, Faculty of Computers and Information, South Valley University, Qena, Egypt \\ ${ }^{2}$ Faculty of Computers and Artificial Intelligence, Cairo University, Giza, Egypt
}

\begin{tabular}{l} 
Article Info \\
\hline Article history: \\
Received Apr 21, 2021 \\
Revised Aug 12, 2021 \\
Accepted Sep 4, 2021 \\
\hline Keywords: \\
CT scan \\
Liver \\
Medical image enhancement \\
Multimodal \\
MRI scan
\end{tabular}

\begin{abstract}
A novel approach for multimodal liver image contrast enhancement is put forward in this paper. The proposed approach utilizes magnetic resonance imaging (MRI) scan of liver as a guide to enhance the structures of computed tomography (CT) liver. The enhancement process consists of two phases: The first phase is the transformation of MRI and CT modalities to be in the same range. Then the histogram of CT liver is adjusted to match the histogram of MRI. In the second phase, an adaptive histogram equalization technique is presented by splitting the CT histogram into two sub-histograms and replacing their cumulative distribution functions with two smooths sigmoid. The subjective and objective assessments of experimental results indicated that the proposed approach yields better results. In addition, the image contrast is effectively enhanced as well as the mean brightness and details are well preserved.
\end{abstract}

This is an open access article under the CC BY-SA license.

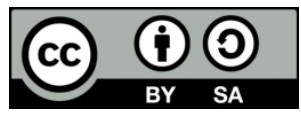

\section{Corresponding Author:}

Ahmed Elaraby

Department of Computer Science, Faculty of Computers and Information, South Valley University

Qena - Safaga Rd, Qena Governorate, Egypt

Email: ahmed.elaraby@svu.edu.eg

\section{INTRODUCTION}

Image enhancement is widely used to improve image clarity [1]-[3]. Medical imaging modalities, for example, includes magnetic resonance imaging (MRI), ultrasound, medical radiation, angiography, and computed tomography (CT) scans. There are several scanning techniques aims at visualizing the human body for diagnostic and treatment purposes. In addition, these modalities are beneficial for patient follow-up, with regards to the progress of the disease state. Medical imaging modalities follow a multidisciplinary approach to obtain the correct diagnosis for a specific patient in order to provide a personalized approach to patient care. These imaging techniques can be applied as non-invasive techniques to view the inside of the human body without any surgical intervention. They can be used to facilitate the diagnosis or treatment of various diseases [4]-[6]. The special resolution required to obtain detailed images of various structures of the human body is a major practical limitation of modern medical imaging techniques. Although, the speed of image acquisition has increased over the past decade, but this does not provide the required sensitivity to express anatomical structure and function, which is dose-limited, among other factors.

Recently, artificial intelligence (AI) based algorithms obtain interesting performance results in diagnosing various diseases from medical images, sometimes even surpassing human-level performance. Exploring how AI might simplify life for the clinicians while keeping the ultimate responsibility for the diagnosis with the human expert may be more valuable in the short term. As AI and medical imaging algorithms gradually improve, they will be able to help less experienced users make better decisions, ultimately improving the quality of patient care. In addition, image quality can be improved with algorithms such as super-resolution 
or smart anti-aliasing, which makes ultrasound scans more detailed and easier to read. AI can also enhance the efficiency by making ultrasound examinations less painful and more beneficial to the doctor than referral to a specialized X-ray ambulance [7].

In the field of biomedical imaging and computer diagnostics, the acquisition and analysis of images using more than one modality (i.e. multimodal) has been a subject of research for many years, since the various modalities include abundant information that can complement each other. As described in one of the multimodal imaging projects for brain tumor segmentation [8], each of the modalities can reveal a unique type of biological information for tumor-induced tissue changes and pose slightly different information processing tasks. Several multimodal imaging techniques, such as the combination of positron emission tomography (PET) and CT, have become the standard of clinical practice. Other methods, such as the simultaneous recording of functional magnetic resonance imaging (fMRI) and electroencephalography (EEG) [9], are widely used for neurobiological research. With the growing volume of multimodal image data and the techniques developed to analyze it, one of the main but largely underestimated problems is that most of the techniques described are conceptually similar, but only deal with a specific imaging problem and a specific scenario. Any classical image fusion method is composed of registration and fusion of features from the registered images [10].

Liver image enhancement techniques are extremely useful in streamlining the planning and navigation phases. These techniques improve visualization of liver and its internal anatomy to help doctors better to diagnose the existence of liver tumour and plan intervention accordingly. Moreover, they make subsequent image-based navigation tasks, such as registration, feature extraction and segmentation, more robust [11]. CT imaging is regarded as the primary tool in diagnosis of various human diseases. However, inferior contrast and imprecise visualization are the drawbacks that limit its utility. CT is frequently preferred over other modalities owing to its quick acquisition time, better ability to capture bony structures and low cost. Keeping human liver into consideration, few structures such as tumours can be better visualized in MR image. While certain vessels are clearly manifested in CT.

Better images can be obtained, if the information from multiple imaging modalities is combined to reconstruct an enhanced image. However, there are few published works in the combination of multiple modalities in the design of guided contrast enhancement approaches [12]-[16]. Among different contrast enhancement approaches, the histogram processing-based approaches get attention [17]-[23]. Multimodality guided image enhancement exploits complimentary information from individual modalities to reconstruct a new image. This reconstructed image improves visualization of the structures of interest, highlights details through establishing reasonable contrast among various areas and enhance fine details of liver anatomy. Preoperative liver image enhancement is the focus of this work. In this paper, we propose a technique to enhance contrast of liver CT image using its corresponding MR image. The image is processed in spatial domain. The impact of our proposed method on the local and global contrast of output image is analysed. Subjective and quantitative assessments of the method is performed using different evaluation matrices.

\section{RESEARCH METHOD}

The main purpose of the presented approach is to enhance the contrast of liver CT to highlight details of liver. CT scans of liver often show low contrast. For enhancement, corresponding high contrast regions of liver MRI image is employed. The datasets for our experimental is consisting of liver CT and MRI liver as DICOM format. The proposed technique has three steps as shown in Figure 1: i) converting CT and MRI images to the same range, ii) the histogram of CT liver image is adjusted to match histogram of reference image MRI liver, and iii) in adaptive histogram modification approach is utilized to enhance the obtain CT liver after adjusting parameters [24]. An adaptive histogram modification approach can be summarized as following: Let's denote $\mathrm{X}$ an input image with size MxN having I possible intensity levels. In this paper assumes I=256.

$$
X=\left[\begin{array}{cccc}
X_{0,0} & X_{0,1} & \cdots & X_{0, N-1} \\
X_{1,0} & X_{1,1} & \cdots & X_{1, N-1} \\
\vdots & \vdots & \ddots & \vdots \\
X_{M-1,0} & X_{M-1,1} & \cdots & X_{M-1, N-1}
\end{array}\right]
$$

Then, let $m$ denote its mean intensity.

$$
m=\frac{\sum_{r=0}^{M-1} \sum_{c=0}^{N-1} X(r, c)}{M N}
$$

Using the mean $m$ as a splitting point, we separate the image histogram $H$ into two sub histograms $H_{L}$ and $H_{U}$ : 


$$
H=H_{L} \cup H_{U}
$$

where,

$$
\begin{aligned}
& H_{L}=\left\{H_{0}, H_{1}, \ldots, H_{m}\right\} \\
& H_{U}=\left\{H_{m+1}, H_{m+2}, \ldots, H_{I-1}\right\}
\end{aligned}
$$

After the splitting, the probability density functions of the two sub histograms are calculated using:

$$
p d f(k)=\left\{\begin{array}{lll}
\frac{H_{L}(k)}{\sum_{n=0}^{m} H_{L}(n)} & \text { if } \quad k \leq m \\
\frac{H_{U}(k)}{\sum_{n=m+1}^{I-1} H_{U}(n)} & \text { if } \quad k>m
\end{array}\right.
$$

where $k \in\{0,1, \ldots, I-1\}$ and it represents the intensity level. Then, the time step to calculate the median is to calculate the cumulative distribution functions for both sub-histograms, which have been packed into (7).

$$
c d f(k)=\left\{\begin{array}{l}
\sum_{n=0}^{k} p d f(n) \quad \text { if } \quad k \leq m \\
\sum_{n=m+1}^{k} p d f(n) \text { if } \quad k>m
\end{array}\right.
$$

Then, the medians of $H_{L}$ and $H_{U}$ denoted by $\mu_{L}$ and $\mu_{U}$, respectively, are computed in (8) and (9) are satisfied.

$$
\begin{array}{lll}
c d f\left(\mu_{L}\right)=0.5 & \text { if } & k \leq m \\
c d f\left(\mu_{U}\right)=0.5 & \text { if } & k>m
\end{array}
$$

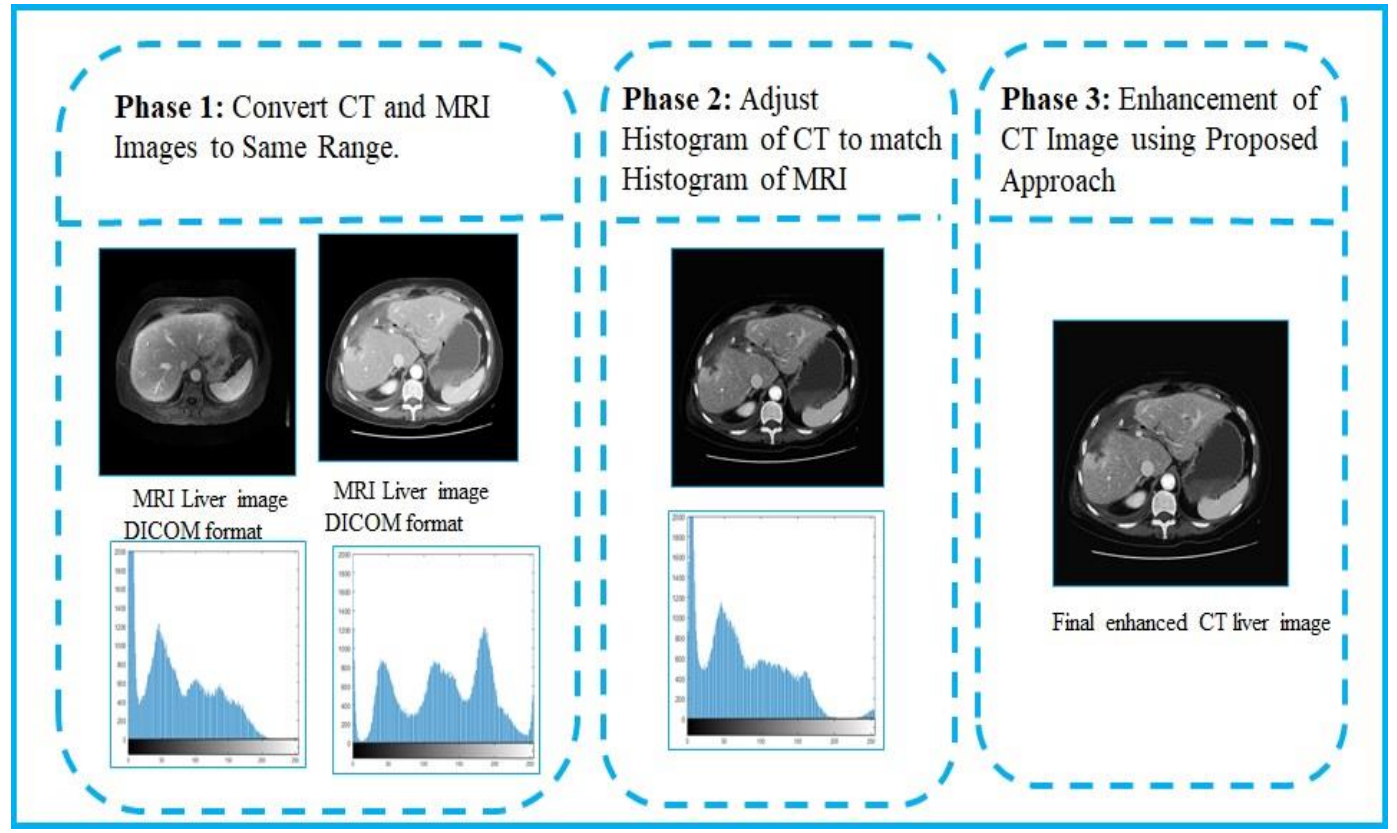

Figure 1. Schematic overview of the proposed methodology

In this module, two parametric non-linear sigmoid functions are created with their origins located on the medians of their corresponding sub-histogram. Their input values $k$ one input or $k$ inputs- need to be normalized, and for this reason we use (10). Therefore, the resulting range of values will fit in the sigmoid desired boundaries and thus, generating $z(k) \in[-5,5] \gamma \geq 1$. This range is convenient because, in (11), the values beyond these limits will be practically 0 or 1 which a parameter $\gamma \geq 1$. In this Figure 1 , we can also get some insight of the graphical from the function corresponding to (11). Therefore, the sigmoid functions described by (11) will take values inside the ranges $[0, m]$ for $k \leq m$ and $[m, I-1]$ for $k>m$. They have 
smooth transitions, hence, avoiding severe affectations by peaks on the histogram, and therefore, abrupt changes on the cumulative distribution function for each sub-histogram are avoided.

$$
\begin{aligned}
& z(k)=\left\{\begin{array}{lll}
\frac{5\left(k-\mu_{L}\right)}{m} \text { if } & k \leq m \\
\frac{5\left(k-\mu_{U}\right)}{I-1-m} \text { if } & k>m
\end{array}\right. \\
& s(k)=\left\{\begin{array}{lll}
\frac{1}{1+e^{-\gamma z(k)}} \text { if } & k \leq m \\
\frac{1}{1+e^{-\gamma z(k)}} \text { if } & k>m
\end{array}\right.
\end{aligned}
$$

Where $\gamma$ is a parameter that controls the smoothness of the sigmoid function.

In our modification scheme, we justify the value of $\gamma$ parameter to $(0.68)$ as the adaptive $\gamma$ can be chosen as:

$$
\gamma=\frac{(128-\mu)}{128}
$$

where $\mu$ is the mean brightness the processed image and for most medical images, their mean brightness is around 40 , hence, $\gamma$ is around 0.68

The last module performs mapping through histogram equalization and stretching. To perform histogram equalization, we apply (13).

$$
u(k)=\left\{\begin{array}{l}
u_{L}=L_{0}+\left(m-L_{0}\right) s(k) \text { if } k \leq m \\
u_{U}=m+\left(L_{f}-m\right) s(k) \text { if } k>m
\end{array}\right.
$$

Where $L_{0}$ and $L_{f}$ represent the desired lower and upper limits, respectively, for the dynamic range of the output image. In this paper $L_{0}=0$ and $L_{f}=I-1$ are supposed.

After obtaining the mappings $u$, we calculate $\alpha_{L}$ and $\alpha_{U}$ values given by (14) and (15).

$$
\begin{aligned}
& \alpha_{L}=\frac{\left(m-L_{0}\right)}{\max \left(u_{L}\right)-\min \left(u_{L}\right)}, \alpha_{U}=\frac{\left(L_{f}-m\right)}{\max \left(u_{U}\right)-\min \left(u_{U}\right)} \\
& T(k)=\left\{\begin{array}{l}
L_{0}+\alpha_{L}\left(u_{L}(k)-\min \left(u_{L}\right)\right) \text { if } k \leq m \\
m+\alpha_{U}\left(u_{U}(k)-\min \left(u_{U}\right)\right) \text { if } k>m
\end{array}\right.
\end{aligned}
$$

Finally, the mapping function from (14) is applied to each pixel of the image $X$, to obtain the enhanced outcome $Y$ as shown in (16).

$$
Y=T(X)
$$

\section{RESULTS AND DISCUSSION}

In this section, we present experimental results obtained as shown in Figure 2. For best of our information, this work is considering the first attempts to apply concept of cross-modality guided quality enhancement of liver image. The performance of the proposed method is evaluated in terms of index of quality improvement using contrast evaluation measures. We use two well-known measures: no reference image quality (NIQE) [25] absolute mean brightness error (AMBE) [26]. These metrics measure different aspects of the image such as image quality, contrast, and brightness preservation, respectively. Nonetheless, it is important to keep in mind that an image could have several artifacts, like mid-gray accumulations, that may compromise the perceptual quality of the image, and still obtain optimum values for these objective measures. Thus, it is desirable to complement the objective assessment with a subjective one, to accurately evaluate the algorithms.

The quantitative measurements are important for further evaluation of the enhancement process. In our experimental, we used the most commonly objective evaluation methods like the absolute mean brightness error (AMBE) and no reference image quality assessment (NIQE). A low score value indicates high perceptual quality. Table 1 indicated the AMBE and NIQE scores of the experimental results. Which shies that bet result when $\gamma=0.5$ which MOS is 4.1 . 
Mean opinion score (MOS) is calculated as the arithmetic mean over single ratings performed by human subjects for a given stimulus in a subjective quality evaluation test: $M O S=\frac{\sum_{n=1}^{N} R_{n}}{N}$, where $R$ is the individual rating matrix for a given stimulus by $N$ subjects. Table 2 indicated MOS scores for the experimental results which show the best results at $\gamma=0.7$ of AMBE and at $\gamma=0.5$ of NIQE.

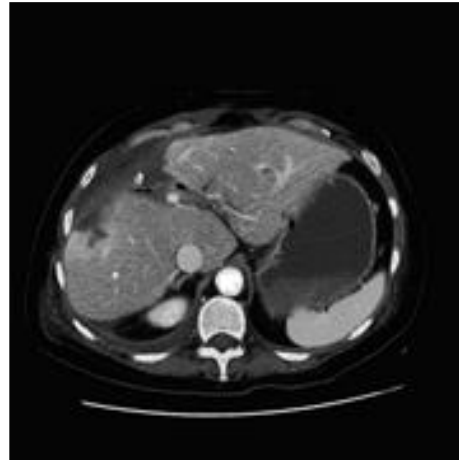

$\gamma=0.2$
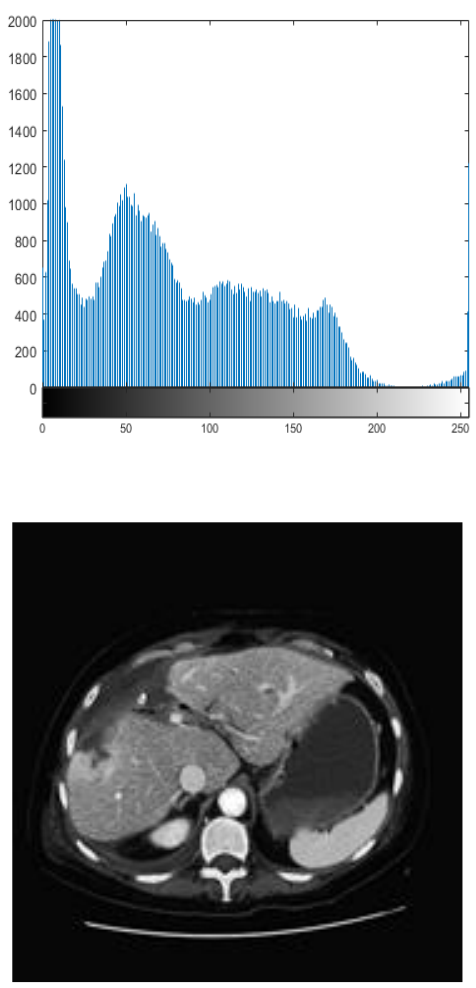

$\gamma=0.5$

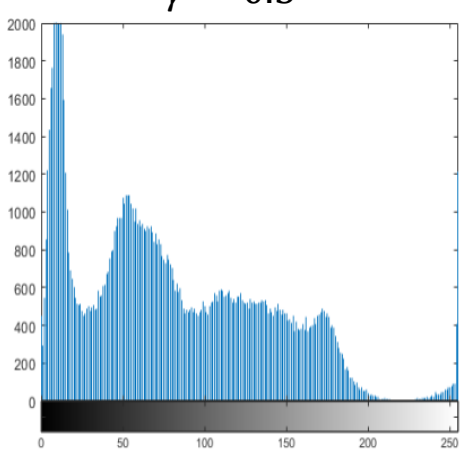

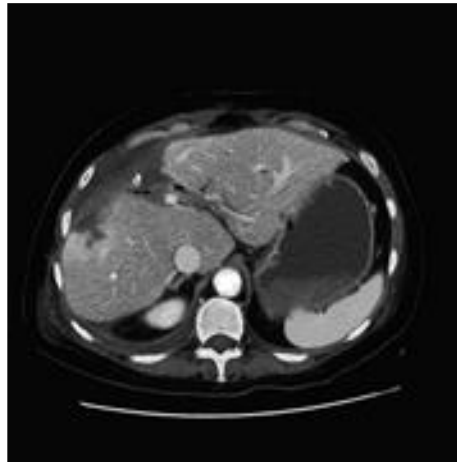

$\gamma=0.3$
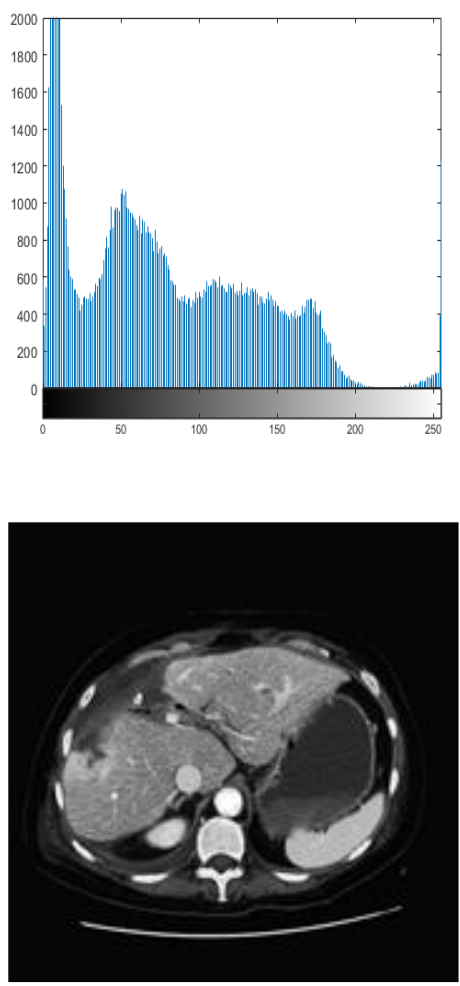

$\gamma=0.6$

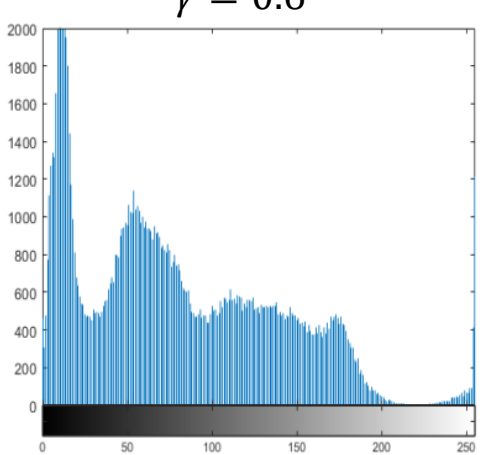

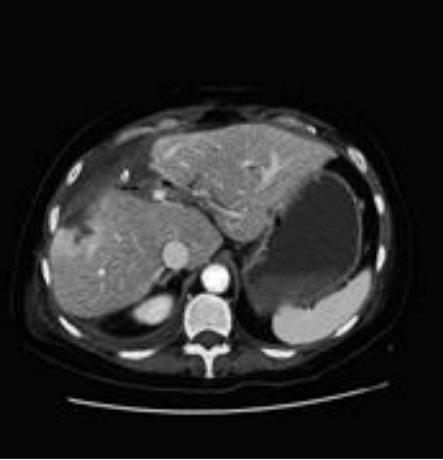

$\gamma=0.4$
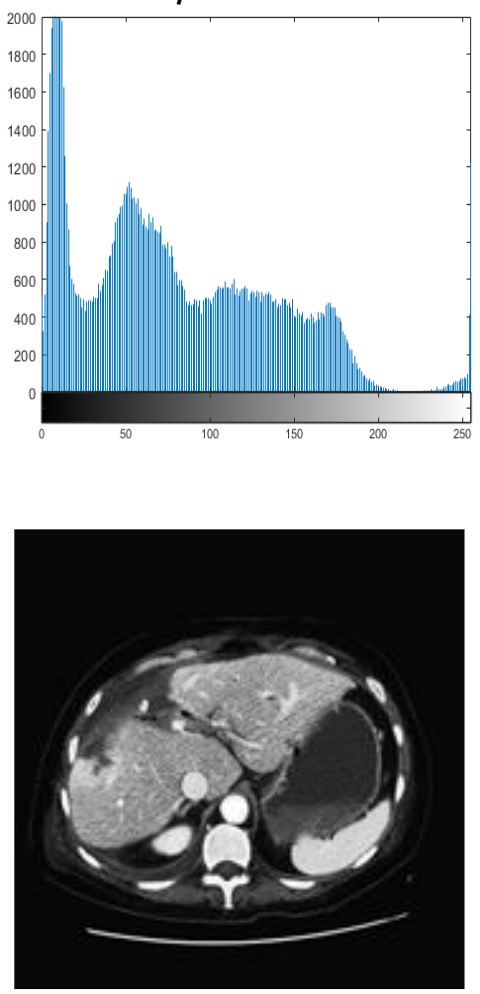

$\gamma=0.7$

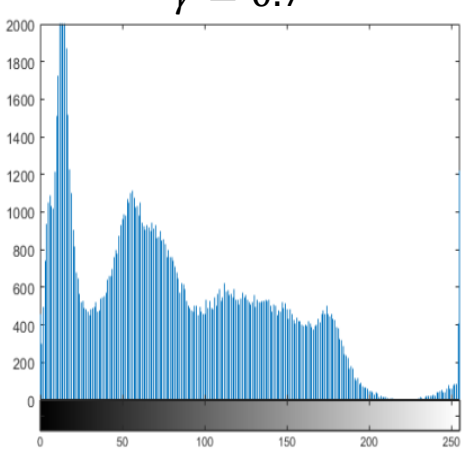

Figure 2. The experimental results of enhanced Liver CT and its histograms 
Table 1. AMBE and NIQE of the experimental results

\begin{tabular}{ccccccc}
\hline Observers & \multicolumn{6}{c}{ Ranking [1:5] } \\
& {$[1$ : Bad-2: Poor-3: Fair-4: Good-:Excellent] } \\
\hline 1 & (a) & (b) & (c) & (d) & (e) & (f) \\
\hline & 3 & 4 & 3 & 4 & 4 & 4 \\
3 & 4 & 5 & 4 & 5 & 4 & 3 \\
4 & 4 & 3 & 4 & 4 & 3 & 5 \\
5 & 4 & 4 & 3 & 5 & 3 & 3 \\
6 & 4 & 3 & 4 & 4 & 4 & 4 \\
7 & 3 & 4 & 4 & 4 & 4 & 5 \\
8 & 3 & 3 & 3 & 3 & 3 & 4 \\
9 & 4 & 4 & 4 & 5 & 4 & 4 \\
10 & 4 & 3 & 5 & 4 & 4 & 3 \\
MOS & 3 & 4 & 4 & 3 & 5 & 4 \\
\hline
\end{tabular}

Table 2. MOS for the experimental results

\begin{tabular}{ccc}
\hline Images & AMBE & NIQE \\
\hline Original CT Liver & - & 6.9478 \\
Enhanced CT liver $(\gamma=0.2)$ & 30.6951 & 4.2249 \\
Enhanced CT liver $(\gamma=0.3)$ & 29.2592 & 4.1509 \\
Enhanced CT liver $(\gamma=0.4)$ & 28.9220 & 4.0806 \\
Enhanced CT liver $(\gamma=0.5)$ & 27.2368 & 4.0554 \\
Enhanced CT liver $(\gamma=0.6)$ & 26.2744 & 4.1002 \\
Enhanced CT liver $(\gamma=0.7)$ & 21.7649 & 4.1829 \\
\hline
\end{tabular}

\section{CONCLUSION}

A multimodality guided enhancement approach of liver is proposed in this paper. The technique focuses on contrast improvement of liver CT. In the domain of medical imaging, such multimodality guided enhancement techniques have not been proposed to the best of our knowledge. The experimental results show that the proposed approach gives better results and highlights fine details of liver CT. Image quality assessment and contrast assessment matrix demonstrate its effectiveness.

\section{REFERENCES}

[1] A. Łoza, D. R. Bull, P. R. Hill, and A. M. Achim, “Automatic contrast enhancement of low-light images based on local statistics of wavelet coefficients," Digital Signal Processing: A Review Journal, vol. 23, no. 6, pp. 1856-1866, Dec. 2013, doi: 10.1016/j.dsp.2013.06.002.

[2] T. Chaira, "An improved medical image enhancement scheme using Type II fuzzy set," Applied Soft Computing Journal, vol. 25, pp. 293-308, Dec. 2014, doi: 10.1016/j.asoc.2014.09.004.

[3] V. Bhateja, M. Misra, and S. Urooj, "Non-linear polynomial filters for edge enhancement of mammogram lesions," Computer Methods and Programs in Biomedicine, vol. 129, pp. 125-134, Jun. 2016, doi: 10.1016/j.cmpb.2016.01.007.

[4] M. S. Lee, C. H. Park, and M. G. Kang, "Edge enhancement algorithm for low-dose X-ray fluoroscopic imaging," Computer Methods and Programs in Biomedicine, vol. 152, pp. 45-52, Dec. 2017, doi: 10.1016/j.cmpb.2017.09.010.

[5] S. Jeevakala and A. Brintha Therese, "Sharpening enhancement technique for MR images to enhance the segmentation," Biomedical Signal Processing and Control, vol. 41, pp. 21-30, Mar. 2018, doi: 10.1016/j.bspc.2017.11.007.

[6] R. Harikumar and B. V. Kumar, "Comprehensive analysis of LPG-PCA algorithms in denoising and deblurring of medical images," International Journal of Imaging Systems and Technology, vol. 23, no. 2, pp. 157-170, May 2013, doi: 10.1002/ima.22049.

[7] T. A. Soomro, L. Zheng, A. J. Afifi, A. Ali, M. Yin, and J. Gao, "Artificial intelligence (AI) for medical imaging to combat coronavirus disease (COVID-19): a detailed review with direction for future research," Artificial Intelligence Review, Apr. 2021, doi: 10.1007/s10462-021-09985-z.

[8] B. H. Menze et al., "The multimodal brain tumor image segmentation benchmark (BRATS)," IEEE Transactions on Medical Imaging, vol. 34, no. 10, pp. 1993-2024, Oct. 2015, doi: 10.1109/TMI.2014.2377694.

[9] M. Czisch, T. C. Wetter, C. Kaufmann, T. Pollmächer, F. Holsboer, and D. P. Auer, "Altered processing of acoustic stimuli during sleep: Reduced auditory activation and visual deactivation detected by a combined fMRI/EEG study," NeuroImage, vol. 16, no. 1, pp. 251-258, May 2002, doi: 10.1006/nimg.2002.1071.

[10] A. P. James and B. V. Dasarathy, "Medical image fusion: A survey of the state of the art," Information Fusion, vol. 19, no. 1, pp. 4-19, Sep. 2014, doi: 10.1016/j.inffus.2013.12.002.

[11] K. Cleary and T. M. Peters, "Image-guided interventions: Technology review and clinical applications," Annual Review of Biomedical Engineering, vol. 12, no. 1, pp. 119-142, Jul. 2010, doi: 10.1146/annurev-bioeng-070909-105249.

[12] X. Zhang, T. Sim, and X. Miao, "Enhancing photographs with near infrared images," Jun. 2008, doi: 10.1109/CVPR.2008.4587825.

[13] Q. Yan et al., "Cross-field joint image restoration via scale map," in Proceedings of the IEEE International Conference on Computer Vision, Dec. 2013, pp. 1537-1544, doi: 10.1109/ICCV.2013.194.

[14] S. Zhuo, X. Zhang, X. Miao, and T. Sim, "Enhancing low light images using near infrared flash images," in Proceedings International Conference on Image Processing, ICIP, Sep. 2010, pp. 2537-2540, doi: 10.1109/ICIP.2010.5652900.

[15] X. Shen, C. Zhou, L. Xu, and J. Jia, "Mutual-structure for joint filtering," in Proceedings of the IEEE International Conference on Computer Vision, Dec. 2015, vol. 2015 Inter, pp. 3406-3414, doi: 10.1109/ICCV.2015.389. 
[16] T. M. Deserno, "Fundamentals of biomedical image processing," Springer Berlin Heidelberg, 2010, pp. 1-51.

[17] R. C. G. and R. E. Woods, Digital Image Processing, 3rd Editio., vol. 19, no. 3. USA: Prentice-Hall, Inc., 2006.

[18] T. Arici, S. Dikbas, and A. Altunbasak, "A histogram modification framework and its application for image contrast enhancement," IEEE Transactions on Image Processing, vol. 18, no. 9, pp. 1921-1935, Sep. 2009, doi: 10.1109/TIP.2009.2021548

[19] S. S. Agaian, B. Silver, and K. A. Panetta, "Transform coefficient histogram-based image enhancement algorithms using contrast entropy," IEEE Transactions on Image Processing, vol. 16, no. 3, pp. 741-758, Mar. 2007, doi: 10.1109/TIP.2006.888338.

[20] C. M. Tsai, "Adaptive local power-law transformation for color image enhancement," Applied Mathematics and Information Sciences, vol. 7, no. 5, pp. 2019-2026, Sep. 2013, doi: 10.12785/amis/070542.

[21] S. C. Huang, F. C. Cheng, and Y. S. Chiu, "Efficient contrast enhancement using adaptive gamma correction with weighting distribution," IEEE Transactions on Image Processing, vol. 22, no. 3, pp. 1032-1041, Mar. 2013, doi: 10.1109/TIP.2012.2226047.

[22] C. Lee, C. S. Kim, and C. Lee, "Contrast enhancement based on layered difference representation of 2D histograms," IEEE Transactions on Image Processing, vol. 22, no. 12, pp. 5372-5384, Dec. 2013, doi: 10.1109/TIP.2013.2284059.

[23] S. C. Huang and C. H. Yeh, "Erratum: Image contrast enhancement for preserving mean brightness without losing image features (Engineering Applications of Artificial Intelligence (2013) 26:5 (1487-1492))," Engineering Applications of Artificial Intelligence, vol. 32, p. 332, Jun. 2014, doi: 10.1016/j.engappai.2014.04.002

[24] E. F. Arriaga-Garcia, R. E. Sanchez-Yanez, and M. G. Garcia-Hernandez, "Image enhancement using Bi-histogram equalization with adaptive sigmoid functions," in CONIELECOMP 2014 - 24th International Conference on Electronics, Communications and Computers, Feb. 2014, pp. 28-34, doi: 10.1109/CONIELECOMP.2014.6808563.

[25] A. Mittal, R. Soundararajan, and A. C. Bovik, "Making a 'completely blind' image quality analyzer," IEEE Signal Processing Letters, vol. 20, no. 3, pp. 209-212, Mar. 2013, doi: 10.1109/LSP.2012.2227726.

[26] S. Der Chen and A. R. Ramli, "Minimum mean brightness error bi-histogram equalization in contrast enhancement," IEEE Transactions on Consumer Electronics, vol. 49, no. 4, pp. 1310-1319, Nov. 2003, doi: 10.1109/TCE.2003.1261234.

\section{BIOGRAPHIES OF AUTHORS}
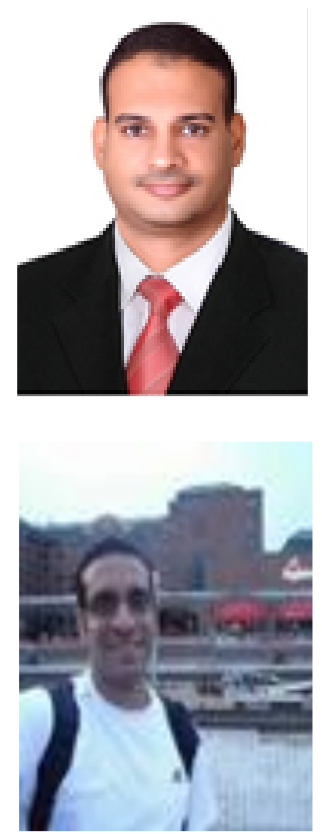

Ahmed Elaraby (iD 81 SC P works as Assistant Professor of Computer Science at South Valley University in Egypt. He received BSc in Computer Science in 2008 from Faculty of Science, South Valley University and worked as a Teaching Assistant at the same Faculty. Meanwhile, he received his MSc degree in the field of computer science in 2012 and his PhD in 2016. In 2012, he was granted for six-months a DAAD research scholarship at HTWG Konstanz University, Germany. In 2013, he was granted for a six-month-research scholarship at Université de Caen Normandie in France. In 2014, he was granted for a 6-month Erasmus Mundus research scholarship at Universitat Politècnica de Valènciain Spain. In 2016, he was granted a one-month research visit at JINR in Russia. In 2018, he granted one-year Post-Doc Fellowship in NTNU Norway. His main research interests are Visual Computing and Biomedical Image Processing. Email: ahmed.elaraby@svu.edi.eg.

Ayman Taha (D) 8d SC P a Marie Curie Career-FIT postdoc research Fellow at the Techn logical University Dublin (TUD), Ireland. He obtained his Ph.D. degree with honors in Computer Information Systems from Cairo University/University of Minnesota through a joint scientific program in 2014. Prior to joining TU Dublin, he served as an Assistant Professor at the Faculty of Computes and Artificial Intelligence at Cairo University, Egypt. Currently, Ayman's research interests are GIS systems, spatial data computing, data mining, categorical data analysis, outliers detection, anomalous events identification, machine learning and deep learning. He received the best PhD Thesis Award at Cairo University in 2014. Email: a.taha@fci-cu.edu.eg. 